\title{
Asociación de la dilatación auricular izquierda con los trastornos del sistema de conducción en pacientes con hipertensión arterial sistémica
}

\author{
Luis Marcelo Miño1,2, *Osmar Antonio Centurión ${ }^{1,2}$, Judith M. Torales ${ }^{1,2}$, Laura B. García ${ }^{1,2}$, Cristina $^{1,2}$ \\ Cáceres $^{1}$, María Paniagua ${ }^{1}$, Oscar A. Lovera ${ }^{1}$, Jorge E. Martínez ${ }^{1}$ \\ ${ }^{1}$ Universidad Nacional de Asunción, Hospital de Clínicas, División de Medicina Cardiovascular. San Lorenzo. \\ Paraguay \\ ${ }^{2}$ Sanatorio Metropolitano, Departamento de Investigación en Ciencias de la Salud. Fernando de la Mora. Paraguay
}

Cómo referenciar este artículo/ How to reference this article:
Miño LM, Centurión OA, Torales JM, García LB, Cáceres $\mathbf{C}$, Paniagua $\mathbf{M}$, et al. Asociación de la dilatación auricular izquierda con los trastornos del sistema de conducción en pacientes con hipertensión arterial sistémica. Mem. Inst. Investig. Cienc. Salud. 2019; 17(3): 20-27

\begin{abstract}
RE S U M E N
La dilatación de la aurícula izquierda (AI) se considera un predictor ecocardiográfico para la remodelación auricular y la fibrilación auricular. Por ende, hemos investigado la correlación existente entre la dilatación de la AI con las arritmias cardíacas y los trastornos del sistema de conducción en pacientes con hipertensión arterial (HTA). En este estudio observacional y prospectivo hemos investigado las variaciones electrocardiográficas, mediciones ecocardiográficas y Holter ECG de 24 hs en pacientes hipertensos y no hipertensos ambulatoria y hospitalizados que acuden a un hospital terciario entre marzo a septiembre del 2018. Se estudiaron 104 pacientes, 65 hipertensos conocidos y 39 no hipertensos como grupo control. El diámetro promedio de la AI fue significativamente mayor $(p=0,03)$ en pacientes hipertensos que los no hipertensos $(37 \pm 8 \mathrm{~mm}$ vs. $34 \pm 5$ $\mathrm{mm})$. Se encontró una asociación significativa entre hipertensión y la aurícula izquierda dilatada (>40 mm) ( $p=0,026$ OR: 3,25 IC95\%: 1,01-11,02). La dilatación de la AI tuvo una especificidad de $73 \%$ y un valor predictivo negativo de $98 \%$ relacionado con la presencia de trastornos del sistema de conducción y arritmias cardiacas en pacientes con HTA. Se encontró asociación entre la hipertensión arterial y la dilatación de la aurícula izquierda. La dilatación de la aurícula izquierda tiene una elevada especificidad y un alto valor predictivo negativo en la detección de la presencia de prolongación del intervalo QT, ensanchamiento del complejo QRS, dispersión de la onda P, y trastornos del sistema de conducción y arritmias cardiacas en pacientes con hipertensión arterial.
\end{abstract}

Palabras clave: Dilatación de la aurícula izquierda, hipertensión arterial, arritmias cardiacas, trastornos del sistema de conducción.

\section{Association of left atrial dilatation with conduction system disorders in patients with systemic arterial hypertension}

\begin{abstract}
A B S T R A C T
Dilation of the left atrium (LA) is considered an echocardiographic predictor for atrial remodeling and atrial fibrillation. Therefore, we have investigated the correlation between dilatation of the LA with cardiac arrhythmias and conduction system disorders in patients
\end{abstract}

* Los autores Luis M. Miño y Osmar A. Centurión contribuyeron equitativamente como primer autor para este trabajo de investigación.

Fecha de recepción: abril 2019. Fecha de aceptación: julio 2019

*Autor correspondiente: Prof. Dr. Osmar Antonio Centurión, MD, PhD, FACC, FAHA

Profesor Titular de Medicina. Facultad de Ciencias Médicas. Universidad Nacional de Asunción-UNA.

Jefe de la División de Medicina Cardiovascular. Hospital de Clínicas. Dirección: Av. Mariscal López e/ Coronel Cazal. San Lorenzo, Paraguay. Teléfono: +(595) 971354444

Email: osmarcenturion@hotmail.com 
with systemic arterial hypertension. In this observational and prospective study we have investigated electrocardiographic variations, echocardiographic measurements and Holter ECG of 24 hours in hypertensive patients who attend a tertiary hospital from March 2018 to September 2018 as outpatients and inpatients. One hundred four patients were studied, 65 known to be hypertensive and 39 non-hypertensive subjects as control group. The diameter of the LA had a mean value of $37 \pm 8$ in hypertensive patients, while in nonhypertensive patients was $34 \pm 5(p=0.03)$. A significant association was found between hypertension and increased diameter of the LA ( $p=0.04$ OR: 2.6 CI $0.88-7.7)$. Dilatation of the LA had a specificity of $73 \%$ and a negative predictive value of $98 \%$ related to the presence of conduction system disorders and cardiac arrhythmias in patients with hypertension. A significant relationship between arterial hypertension and dilatation of the left atrium was observed. The dilatation of the left atrium has a high specificity and a high negative predictive value in the detection of the presence of prolongation of the QT interval, widening of the QRS complex, dispersion of the P wave, and disorders of the conduction system and cardiac arrhythmias in patients with hypertension.

Keywords: Dilatation of the left atrium, arterial hypertension, cardiac arrhythmias, conduction system disorders.

\section{INTRODUCCION}

La prevalencia de hipertensión arterial (HTA) es alta en los Estados Unidos y en todo el mundo, y el tratamiento de la hipertensión es la razón más común para las visitas al consultorio médico de adultos en los Estados Unidos y para el uso de medicamentos recetados $^{(1-6)}$. Además, aproximadamente la mitad de los individuos hipertensos no tienen un control adecuado de la presión arterial. La HTA tiene la característica de que siendo una enfermedad es también un factor de riesgo para las patologías cardiovasculares, cerebrovasculares y renales. Asimismo, puede producir afectaciones vasculares periféricas y de la retina ${ }^{(7-10)}$.

La educación y prevención de riesgos de la HTA conforman las medidas sanitarias más importantes, universales y menos costosas. El adecuado control y seguimiento de la presión arterial es un desafío para todos los países y debe ser prioridad de las instituciones de salud, población y gobiernos; siendo considerada una de las afecciones más frecuentes con importantes repercusiones en el orden personal, económico y sanitario ${ }^{(11-16)}$. Por ello, las acciones educativas asociados a esta patología constituyen pilares de actuación en su enfrentamiento.

Se estima que 691 millones de personas padecen HTA. De los 15 millones de muertes causadas por enfermedades circulatorias, 7,2 millones son enfermedades coronarias y 4,6 millones por enfermedad vascular encefálica, siendo la HTA factor de riesgo presente en la mayoría de ellas. En la mayor parte de las regiones existe una prevalencia del 15 al $30 \%$ de la enfermedad ${ }^{(17-24)}$.

La dilatación de la aurícula izquierda por ecocardiografía puede ser un hallazgo diagnóstico y que puede aportar datos de gran valor a la hora de determinar el pronóstico de la HTA. La disfunción diastólica que produce la HTA favorece la dilatación de la aurícula izquierda y el posterior desarrollo de la fibrilación auricular ${ }^{(25)}$.

Actualmente se posee escasa estadística nacional acerca de ciertos hallazgos en la HTA por los métodos auxiliares de diagnóstico. Por ejemplo, las alteraciones que pueden ser encontradas en el electrocardiograma (ECG) que es el estudio más accesible en el sistema de salud de nuestro país, así como en el ECG ambulatorio Holter de 24 hs, y en la ecocardiografía. Por lo tanto, el objetivo de este estudio fue analizar la relación existente entre la dilatación de la aurícula izquierda medida por ecocardiografía bidimensional y el desarrollo de arritmias cardiacas y trastornos del sistema de conducción en pacientes con hipertensión arterial.

\section{MATERIAL Y METODOS}

Estudio observacional - prospectivo de corte trasversal con componentes analíticos y muestreo no probabilístico de conveniencia en el que se estudiaron pacientes ambulatorios e internados que se realizaron sus estudios de electrocardiograma, Holter 24 horas y ecocardiografía en el Departamento de Cardiología del Hospital de Clínicas entre los meses de marzo y septiembre del 2018.

Los criterios de inclusión fueron: Pacientes mayores de 18 años que acudieron al Servicio de Cardiología del Hospital de Clínicas a quienes se les realiza ECG, Holter y 
Ecocardiografía Doppler en el periodo de marzo del 2018 a septiembre del 2018, con consentimiento informado. Pacientes internados en diferentes servicios del Hospital de Clínicas (cardiología, medicina interna, neumología, cirugía, ginecoobstetricia) a quienes se les realiza ECG, Holter y ecocardiografía Doppler con su consentimiento. Se excluyeron pacientes portadores de fibrilación auricular permanente y flutter auricular.

Se realizó ECG de 12 derivaciones, Holter de 24 horas y Ecocardiografía Doppler transtorácica.

Las variables analizadas fueron la edad y sexo, antecedentes patológicos personales, tratamiento, ECG (valor de la onda P, Pmáx, Pmin, dispersión de la onda P, QRS, QT, Qtx), Holter (FC máx, FC min, Promedio de FC, Extrasístoles supraventriculares, duplas auriculares, taquiarritmias auriculares, extrasístoles ventriculares, duplas ventriculares, presencia de taquiarritmias ventriculares, bradiarritmias), y ecocardiograma (diámetro de aurícula izquierda, área de aurícula izquierda).

Se completó una planilla de elaboración propia en el programa Microsoft Excel 2015, que contenía los datos del electrocardiograma de 12 derivaciones, Holter y ecocardiograma Doppler transtorácica.

Las variables derivadas del valor de la onda $\mathrm{P}$ fueron consideradas normales aquellas cuya duración era menor a 120 ms y aumentada en aquellos pacientes que cuyo valor era mayor a 120 ms, la dispersión de $\mathrm{P}$ fue considerada normal si su valor era menor a 39 y aumentada si era igual o mayor que 39. La variable QTC se consideró normal cuando su valor era menor o igual a $440 \mathrm{~ms}$ en varones y $450 \mathrm{~ms}$ en mujeres, caso contrario fueron consideradas aumentadas. En cuanto a la dispersión del QT se consideró normal aquellos cuyos valores eran menores o iguales a $68 \mathrm{~ms}$ caso contrario se consideraron aumentados. La duración del complejo QRS se consideró normal entre 80 y 120 ms y aumentado si la duración era mayor a 120 ms. El diámetro del ventrículo izquierdo en diástole se consideró normal si se encontraba entre 42 y $49 \mathrm{~mm}$ caso contrario se consideró anormal. El diámetro de la aurícula izquierda se utilizó como punto de corte 40 $\mathrm{mm}$, en el área de la aurícula izquierda se utilizó como punto de corte $30 \mathrm{~cm}^{2}$. El diámetro sistólico del ventrículo izquierdo se consideró normal entre 30 y $40 \mathrm{~mm}$. Para la fracción de eyección se utilizó como punto de corte $55 \%$.

\section{Análisis estadísticos}

Los datos fueron cargados en una hoja de cálculo de Microsoft Excel hallándose la frecuencia de variables cualitativas y las medidas de tendencia central de las variables cualitativas. Para el análisis estadístico fueron dicotomizadas las variables cuantitativas y se usó el Chi Cuadrado como estadístico de prueba usándose el paquete estadístico Epi Info 7.

\section{Consideraciones éticas}

El estudio fue realizado de acuerdo a una buena práctica clínica, conforme a la Declaración de Helsinki, aprobado por el comité de ética local, y con el respectivo consentimiento informado del paciente. Además, los datos se manejaron en forma confidencial, en ningún caso se publicaron los nombres de los participantes.

\section{RESULTADOS}

Fueron estudiados 104 pacientes, cuya media de edad fue de 57,5 $\pm 17,3$ años, 54 de los pacientes fueron de sexo masculino y 50 de sexo femenino; 65 pacientes fueron hipertensos conocidos y 39 personas no hipertensas (Tabla 1).

Tabla 1: Distribución por sexo de la edad y presencia de hipertensión arterial

\begin{tabular}{llll}
\hline & Femenino & $\begin{array}{c}\text { Sexo } \\
\text { Masculino }\end{array}$ & Total \\
\hline Edad & & & \\
$18-30$ & $6(46,2 \%)$ & $7(53,8 \%)$ & 13 \\
$31-42$ & $4(66,7 \%)$ & $2(33,3 \%)$ & 6 \\
$43-54$ & $9(47,4 \%)$ & $10(52,6 \%)$ & 19 \\
$55-66$ & $14(41,2 \%)$ & $20(58,8 \%)$ & 34 \\
$67-78$ & $8(38,1 \%)$ & $13(61,9 \%)$ & 21 \\
$79-91$ & $7(63,6 \%)$ & $4(36,4 \%)$ & 11 \\
Hipertensión arterial & $29(58 \%)$ & $36(66,7 \%)$ & 65 \\
\hline
\end{tabular}


El diámetro promedio de la AI fue significativamente mayor $(p=0,03)$ en los 65 pacientes hipertensos que los 39 pacientes no hipertensos ( $37 \pm 8 \mathrm{~mm}$ vs. $34 \pm 5 \mathrm{~mm}$ ). Se encontró una asociación significativa entre hipertensión y la aurícula izquierda dilatada $(>40 \mathrm{~mm})(p=0,026$ OR: 3,25 IC95\%: 1,01-11,02). (Tabla 2).

De los 39 pacientes no hipertensos, 5 presentaron una AI dilatada, con una media de $34 \pm 5$. El índice de volumen de la AI estaba aumentado en 8 de los hipertensos con una media de $16 \pm 6 \mathrm{~cm}^{2}$. Y en 6 de los no hipertensos con una media de $12 \pm 6 \mathrm{~cm}^{2}$. No se halló asociación significativa entre hipertensión y el índice de volumen de la aurícula izquierda $(p=0,45$ OR: 0,9 IC $0,29-3,1)$.

Los 65 pacientes hipertensos presentaban una media de dispersión de la onda $P$ de $37 \pm 8 \mathrm{~ms}$ en comparación a $27 \pm 13 \mathrm{~ms}$ en los 39 pacientes no hipertensos. Se encontró una asociación significativa entre HTA y la dispersión de onda $P(p=0,012$ OR: 2,81 IC $1,15-6,94)$. (Tabla 2)

No se constataron taquiarritmias supraventriculares (FA y Flutter) en el ECG ambulatorio Holter de 24 hs en los pacientes hipertensos, en cambio se observó FA en 1 paciente y flutter auricular en 3 pacientes del grupo sin HTA.

De los 65 pacientes hipertensos 13 presentaban un complejo QRS aumentado ( $>120$ ms) y 52 presentaban un complejo QRS estrecho, presentando un promedio de duración del complejo QRS de $101 \pm 19$ ms. En cambio, de los pacientes normotensos (39 pacientes), 7 presentaban un complejo QRS ensanchado, y 32 lo tenían en rango normal, con un promedio del complejo QRS de $95 \pm 21 \mathrm{~ms}$. No se hallaron asociaciones significativas entre hipertensión y alteraciones de la duración del QRS ( $p=0,45 ;$ OR: 1,12 IC95\%: 0,4-3,13).

De los 65 pacientes hipertensos, 35 eran varones, de los cuales 5 pacientes $(7,6 \%)$ presentaron QT corregido prolongado ( $\geq 450 \mathrm{~ms}$ ) con una media de $486,8 \mathrm{~ms} ; 2$ pacientes presentaron Intervalos QT menor a $390 \mathrm{~ms}$, con una media de $380 \mathrm{~ms}$. De las 30 mujeres hipertensas, 4 presentaron intervalos QT aumentado (mayor o igual a $460 \mathrm{~ms}$ ), con una media de 471,75 ms y 2 presentan intervalo QT disminuido con una media de $379 \mathrm{~ms}$.

De los 39 pacientes normotensos, 20 eran varones, de los cuales $7(17,9 \%)$ presentaban un intervalo QT aumentado ( $\geq 450 \mathrm{~ms}$ ) con una media de $487,8 \mathrm{~ms}$ y 2 presentaron un intervalo QT menor a $390 \mathrm{~ms}$ con una media de $387,5 \mathrm{~ms}$. De las 19 mujeres no hipertensas ninguna presentó un intervalo QT prolongado, 4 de ellas presentaron un intervalo QT menor a 390 ms con una media de 368 ms. No se encontró una asociación significativa entre HTA y QTc prolongado.

Tabla 2: Datos electrocardiográficos y ecocardiográficos.

\begin{tabular}{|c|c|c|c|c|}
\hline & $\begin{array}{l}\text { Hipertensos } \\
(n=65)\end{array}$ & $\begin{array}{l}\text { Normotensos } \\
(\mathrm{n}=39)\end{array}$ & Valor $\mathbf{p}$ & OR (IC95\%) \\
\hline Diámetro auricular & & & 0,026 & $3,25(1,01-11,02)$ \\
\hline Aumentado & $21(32 \%)$ & $5(12,8 \%)$ & & \\
\hline Normal & $44(68 \%)$ & $34(87,2 \%)$ & & \\
\hline Dispersión de onda P & & & 0,012 & $2,81(1,15-6,94)$ \\
\hline Aumentada & $43(66,1 \%)$ & $16(41 \%)$ & & \\
\hline Normal & $22(33,9 \%)$ & $23(49 \%)$ & & \\
\hline Intervalo QT corregido & & & 0,13 & $0,53(0,21-1,33)$ \\
\hline $\begin{array}{l}\text { Aumentado } \\
\text { Normal }\end{array}$ & $\begin{array}{l}19(29,2 \%) \\
46(70,8 \%)\end{array}$ & $\begin{array}{l}17(43,6 \%) \\
22(65,4 \%)\end{array}$ & & \\
\hline
\end{tabular}

\section{Relación entre la dilatación de la aurícula izquierda y otros parámetros específicos en la hipertensión arterial}

Si analizamos la relación entre la dilatación de la aurícula izquierda con los trastornos del sistema de conducción y las arritmias cardiacas en los pacientes con hipertensión arterial podemos observar que de los 19 (29\%) pacientes hipertensos que tenían una dilatación de aurícula izquierda ( $>40 \mathrm{~mm}), 2$ pacientes tenían trastornos del sistema de conducción y arritmias cardiacas y 17 pacientes no la presentaron. Sin embargo, de los 46 (71\%) pacientes hipertensos que tenían un diámetro de la aurícula izquierda normal ( $\leq 40$ $\mathrm{mm}), 1$ paciente tenía trastornos del sistema de conducción y arritmias cardiacas, y 45 pacientes no la presentaron. Estos hallazgos determinan que la dilatación de la aurícula izquierda posee una alta especificidad, un muy elevado valor predictivo negativo y una 
moderada sensibilidad relacionado con la presencia de trastornos del sistema de conducción y arritmias cardiacas en pacientes con hipertensión arterial (Tabla 3).

Por otro lado, si realizamos un análisis de la relación entre la dilatación de la aurícula izquierda con la duración del complejo QRS en los pacientes con hipertensión arterial podemos observar que de los $19(29 \%)$ pacientes hipertensos que tenían una dilatación de la aurícula izquierda (>40 mm), 5 pacientes tenían un complejo QRS ensanchado y 14 pacientes presentaban un complejo QRS angosto. Sin embargo, de los $46(71 \%)$ pacientes hipertensos que tenían un diámetro de la aurícula izquierda normal ( $\leq 40 \mathrm{~mm}), 6$ pacientes tenían complejo QRS ensanchado, y 40 pacientes presentaban un complejo QRS angosto. Estos hallazgos determinan que la dilatación de la aurícula izquierda posee una alta especificidad, un muy elevado valor predictivo negativo y una moderada sensibilidad relacionado con la presencia de ensanchamiento del complejo QRS del electrocardiograma convencional (Tabla 3).

Con relación al análisis de la relación entre la dilatación de la aurícula izquierda con la duración del intervalo QT del electrocardiograma en los pacientes con hipertensión arterial podemos observar que de los $19(29 \%)$ pacientes hipertensos que tenían una dilatación de la aurícula izquierda (>40 mm), 7 pacientes tenían un intervalo QT corregido prolongado y 12 pacientes presentaban un intervalo QT corregido normal. Sin embargo, de los 46 (71\%) pacientes hipertensos que tenían un diámetro de la aurícula izquierda normal ( $\leq 40$ $\mathrm{mm}), 11$ pacientes tenían un intervalo QT corregido prolongado, y 35 pacientes presentaban un intervalo QT corregido normal. Estos hallazgos determinan que la dilatación de la aurícula izquierda posee una alta especificidad y un elevado valor predictivo negativo relacionado con la presencia de prolongación del intervalo QT en el electrocardiograma convencional (Tabla 3).

Con relación al análisis de la relación entre la dilatación de la aurícula izquierda con la dispersión de la onda $\mathrm{P}$ en los pacientes con hipertensión arterial podemos observar que de los 19 (29\%) pacientes hipertensos que tenían una dilatación de la aurícula izquierda (>40 mm), ningún paciente presentó una dispersión de la onda $P$ alterada y 19 pacientes presentaron una dispersión de la onda $\mathrm{P}$ normal. Sin embargo, de los 46 (71\%) pacientes hipertensos que tenían un diámetro de la aurícula izquierda normal ( $\leq 40 \mathrm{~mm}), 5$ pacientes tenían una dispersión de la onda $\mathrm{P}$ alterada y 41 pacientes presentaban una dispersión de la onda $\mathrm{P}$ normal. Estos hallazgos determinan que la dilatación de la aurícula izquierda posee una alta especificidad y un elevado valor predictivo negativo relacionado con la presencia de la dispersión de la onda P (Tabla 3).

Tabla 3: Relación entre la dilatación de la aurícula izquierda con otros parámetros

\begin{tabular}{lllll}
\hline Relación & TSC y Arritmias & Complejo QRS & Intervalo QT & Dispersión Onda P \\
Sensibilidad & $67 \%$ & $45 \%$ & $39 \%$ & $0 \%$ \\
Especificidad & $73 \%$ & $74 \%$ & $74 \%$ & $68 \%$ \\
V. Predictivo P. & $11 \%$ & $26 \%$ & $37 \%$ & $0 \%$ \\
V. Predictivo N. & $98 \%$ & $87 \%$ & $76 \%$ & $89 \%$ \\
Prevalencia & $0.4 \%$ & $17 \%$ & $28 \%$ & $0.7 \%$ \\
\hline
\end{tabular}

\section{DISCUSIÓN}

La hipertensión arterial es una de las enfermedades crónicas más prevalentes en el mundo actual y en nuestro país, constituyendo un motivo de consulta frecuente en la Cátedra y Servicio de Cardiología del Hospital de Clínicas ${ }^{(26-30)}$. No solo la hipertensión es frecuente en el Hospital de Clínicas sino que también los daños crónicos que causa esta si no es tratada adecuadamente como lo es la enfermedad renal, la cardiopatía isquémica, los accidentes cerebrovasculares ${ }^{(31-35)}$. Esto puede ser debido al estilo sedentario de vida en nuestro país y las artes culinarias que se caracterizan por su alto contenido de sodio en las preparaciones.

La hipertensión en el presente estudio afectó de manera casi igual a ambos sexos incrementando su incidencia entre la cuarta y sexta década de vida que es similar a lo esperado según la bibliografía consultada(1-5). Cabe destacar que en la presente investigación se hallaron asociaciones significativas entre la presencia de hipertensión arterial y dilatación de la aurícula izquierda. Además, se observó una relación con la dispersión de la onda $\mathrm{P}$ al electrocardiograma. Los pacientes hipertensos tenían 3 veces 
más probabilidad de presentar una dispersión de la onda $p$ aumentada al ECG de 12 derivaciones que el grupo control. También se encontró una asociación significativa entre la hipertensión y el intervalo QTC presentando los pacientes hipertensos 2,4 veces más probabilidad de presentar un intervalo QTC alterado.

Los pacientes hipertensos presentaron 2,6 veces más probabilidad de presentar un diámetro de la aurícula izquierda aumentado siendo este un hallazgo significativo; no obstante, en el presente estudio no se encontró una asociación significativa entre la hipertensión y la superficie de la aurícula izquierda. Sin embargo, los hipertensos presentaron 1,1 veces menos probabilidad de presentar una superficie auricular aumentada.

Dado los hallazgos de la dispersión de la onda $\mathrm{P}$ que está asociada a una mayor incidencia de arritmias cardiacas ${ }^{(36-42)}$, resultaría natural esperar que en los hipertensos se encuentre también aumentado el diámetro y la superficie auricular izquierda. A pesar de que se encontró una asociación significativa entre el aumento de diámetro de la aurícula izquierda y la hipertensión no se observaron asociaciones significativas con la superficie auricular, probablemente debido al escaso número de pacientes lo cual es una limitación de este estudio. También otra limitación del presente estudio es que la muestra no fue pareada entre pacientes hipertensos y normotensos lo que podría restar potencia estadística a los hallazgos realizados.

En conclusión, existe una relación significativa entre la hipertensión arterial y la dilatación de la aurícula izquierda. La dilatación de la aurícula izquierda tiene una elevada especificidad y un alto valor predictivo negativo en la detección de la presencia de prolongación del intervalo QT, ensanchamiento del complejo QRS, dispersión de la onda $\mathrm{P}$, y trastornos del sistema de conducción y arritmias cardiacas en pacientes con hipertensión arterial sistémica.

\section{REFERENCIAS BIBLIOGRAFICAS}

1. Williams B, Mancia G, Spiering W, Agabiti Rosei $E$, Azizi M, Burnier $M$, et al. ESC/ESH Guidelines for the management of arterial hypertension. Eur Heart J 2018; 39: 3021-104.

2. Lewington $S$, Clarke $R$, Qizilbash N, Peto $\mathrm{R}$, Collins R. Prospective Studies Collaboration. Age-specific relevance of usual blood pressure to vascular mortality: a meta-analysis of individual data for one million adults in 61 prospective studies. Lancet. 2002; 360(9349): 1903-13.

3. Muntner $\mathrm{P}$, Carey RM, Gidding $\mathrm{S}$, Jones DW, Taler SJ, Wright JT Jr, et al. Potential US Population Impact of the 2017 ACC/AHA High Blood Pressure Guideline. Circulation 2018; 137(2): 10918.

4. Myers MG. A proposed algorithm for diagnosing hypertension using automated office blood pressure measurement. J Hypertens. 2010;28(4):703-8.

5. Forman JP, Stampfer MJ, Curhan GC. Diet and lifestyle risk factors associated with incident hypertension in women. JAMA. 2009; 302(4):401-11.

6. Sonne-Holm S, Sørensen TI, Jensen G, Schnohr P. Independent effects of weight change and attained body weight on prevalence of arterial hypertension in obese and non-obese men. BMJ. 1989; 299 (6702): 767-70.

7. Staessen JA, Wang J, Bianchi G, Birkenhäger WH. Essential hypertension. Lancet. 2003; 361(9369):1629-41.
8. Wang $\mathrm{N}-\mathrm{Y}$, Young $\mathrm{JH}$, Meoni LA, Ford DE, Erlinger TP, Klag $\mathrm{MJ}$. Blood pressure change and risk of hypertension associated with parental hypertension: the Johns Hopkins Precursors Study. Arch Intern Med. 2008; 168(6):643-8.

9. Carnethon MR, Evans NS, Church TS, Lewis CE, Schreiner PJ, Jacobs DR, et al. Joint associations of physical activity and aerobic fitness on the development of incident hypertension: coronary artery risk development in young adults. Hypertension. julio de 2010;56(1):49-55.

10. Lorell BH, Carabello BA. Left ventricular hypertrophy: pathogenesis, detection, and prognosis. Circulation. 2000;102(4):4709.

11. Vakili BA, Okin PM, Devereux RB. Prognostic implications of left ventricular hypertrophy. Am Heart J. marzo de 2001;141(3):334-41.

12. Levy D, Larson MG, Vasan RS, Kannel WB, Ho KK. The progression from hypertension to congestive heart failure. JAMA. 22 de mayo de 1996; 275(20):1557-62.

13. Staessen JA, Fagard $R$, Thijs $L$, Celis $H$, Arabidze GG, Birkenhäger $W H$, et al. Randomised double-blind comparison of placebo and active treatment for older patients with isolated systolic hypertension. The Systolic Hypertension in Europe (Syst-Eur) Trial Investigators. Lancet. 1997; 350 (9080):757-64.

14. Thrift AG, McNeil JJ, Forbes A, Donnan GA. Risk factors for cerebral hemorrhage in the era of well-controlled hypertension. 
Miño, et al Asociación de la dilatación auricular izquierda con los trastornos del sistema de conducción ...

Melbourne Risk Factor Study (MERFS) Group. Stroke. noviembre de 1996;27(11):2020-5.

15. Wilson PW. Factores de riesgo establecidos y enfermedad arterial coronaria: el estudio de Framingham. Am J Hypertens 1994; 7: 7S.

16. Coresh J, Wei GL, McQuillan G, Brancati FL, Levey AS, Jones C, et al. Prevalence of high blood pressure and elevated serum creatinine level in the United States: findings from the third National Health and Nutrition Examination Survey (19881994). Arch Intern Med. 2001; 161(9):1207-16.

17. Reboussin DM, Allen NB, Griswold ME, Guallar E, Hong Y, Lackland DT, et al. Systematic Review for the 2017 ACC/AHA/AAPA/ABC/ACPM/AGS/APhA/AS $\mathrm{H} / \mathrm{ASPC} / \mathrm{NMA}$ /PCNA Guideline for the Prevention, Detection, Evaluation, and Management of High Blood Pressure in Adults: A Report of the American College of Cardiology/American Heart Association Task Force on Clinical Practice Guidelines. J Am Coll Cardiol. 2018; 71(19):2176-98.

18. Franklin SS, Larson MG, Khan SA, Wong $N D$, Leip EP, Kannel WB, et al. Does the relation of blood pressure to coronary heart disease risk change with aging? The Framingham Heart Study. Circulation. 2001;103(9):1245-9.

19. Eckel RH, Jakicic JM, Ard JD, de Jesus JM, Houston Miller N, Hubbard VS, et al. AHA/ACC guideline on lifestyle management to reduce cardiovascular risk: a report of the American College of Cardiology/American Heart Association Task Force on Practice Guidelines. J Am Coll Cardiol. 2014; 63(25 Pt B): 2960-84.

20. Mancia G, Fagard R, Narkiewicz K, Redón J, Zanchetti A, Böhm M, et al. ESH/ESC Guidelines for the management of arterial hypertension: the Task Force for the management of arterial hypertension of the European Society of Hypertension (ESH) and of the European Society of Cardiology (ESC). J Hypertens. 2013;31(7):1281-357.

21. Daskalopoulou SS, Rabi DM, Zarnke KB, Dasgupta K, Nerenberg K, Cloutier L, et al. The 2015 Canadian Hypertension Education Program recommendations for blood pressure measurement, diagnosis, assessment of risk, prevention, and treatment of hypertension. Can J Cardiol. 2015; 31(5):549-68.

22. Chobanian AV, Bakris GL, Black HR. El Séptimo Informe del Comité Nacional Conjunto sobre Prevención, Detección, Evaluación y Tratamiento de la Presión Arterial Alta: el informe JNC 7. JAMA 2003; 289: 2560.

23. Mancia G, De Backer G, Dominiczak A, et al. Directrices de 2007 para el manejo de la hipertensión arterial: el Grupo de trabajo para el manejo de la hipertensión arterial de la Sociedad Europea de Hipertensión (ESH) y de la Sociedad Europea de Cardiología (ESC). J Hypertens 2007; 25: 1105.

24. Centurión OA, Aquino-Martinez NJ, Torales-Salinas JMLA. Left Atrial Volume Index as a Clinical Marker for Atrial Fibrillation and Predictor of Cardiovascular Outcomes. J Cardiol Curr Res 2016;6(5):00223. DOI: 10.15406/jccr.2016.06.00223

25. Ramirez MO, Pino CT, Furiasse LV, Lee AJ, Fowkes FG. Paraguayan National Blood Pressure Study: prevalence of hypertension in the general population. J Hum Hypertens. 1995; Nov:9(11):891-7.

26. World Health Organization. STEPwise approach to surveillance (STEPS) Paraguay leaflet. Disponible en: http://www.who.int/chp/steps/2011_STEP S_Paraguay_leaflet.pdf.

27. Organización Panamericana de la Salud. Indicadores básicos de salud. Paraguay 2015. Washington, DC: OPS; 2015. Disponible en http://www.paho.org/par/index.php?optio $\mathrm{n}=$ com_content\&view $=$ article\&id $=25$ : indic adoresbasicos-salud.

28. James PA, Oparil S, Carter BL, Cushman WC, Dennison-Himmelfarb C, Handler J, et al. 2014 evidence-based guideline for the management of high blood pressure in adults: report from the panel members appointed to the Eighth Joint National Committee (JNC 8). JAMA. 2014; 311(5):507-20.

29. Elmer PJ, Obarzanek E, Vollmer WM, Simons-Morton D, Stevens VJ, Young DR, et al. Effects of comprehensive lifestyle modification on diet, weight, physical fitness, and blood pressure control: 18month results of a randomized trial. Ann Intern Med. 2006; 144(7):485-95.

30. Law MR, Morris JK, Wald NJ. Use of blood pressure lowering drugs in the prevention of cardiovascular disease: meta-analysis of 147 randomised trials in the context of expectations from prospective epidemiological studies. BMJ. 19 de mayo de 2009;338:b1665

31. Webb AJS, Fischer $U$, Mehta $Z$, Rothwell PM. Effects of antihypertensive-drug class on interindividual variation in blood pressure and risk of stroke: a systematic review and meta-analysis. Lancet. 13 de marzo de 2010;375(9718):906-15.

32. O'Mailia JJ, Sander GE, Giles TD. Nifedipine-associated myocardial ischemia or infarction in the treatment of hypertensive urgencies. Ann Intern Med. $1987 ; 107(2): 185-6$.

33. Grossman E, Messerli FH, Grodzicki T, Kowey P. Should a moratorium be placed on sublingual nifedipine capsules given for hypertensive emergencies and 
Miño, et al Asociación de la dilatación auricular izquierda con los trastornos del sistema de conducción ...

pseudoemergencies? JAMA. 23 de octubre de $1996 ; 276(16): 1328-31$.

34. Schmieder RE, Rockstroh JK, Messerli FH. Antihypertensive therapy. To stop or not to stop? JAMA. 1991;265(12):1566-71.

35. Nelson MR, Reid CM, Krum $H$, Ryan $P$, Wing LMH, McNeil JJ, et al. Short-term predictors of maintenance of normotension after withdrawal of antihypertensive drugs in the second Australian National Blood Pressure Study (ANBP2). Am J Hypertens. 2003;16(1):39-45.

36. Centurión OA, García LB, Marecos A, Torales J, Scavenius K, Miño L, Sequeira O. P-Wave Morphology, Amplitude, Duration and Dispersion in Atrial Arrhythmias. J Cardiol Therap 2018;6:1-6.

37. Centurión $O A$, Aquino N, Torales J, Scavenius K, Miño L, Sequeira O. P Wave Duration and Dispersion as a Useful Conventional Electrocardiographic Marker for Atrial Fibrillation Prediction. J Cardiol Curr Res 2017; 8(3): 00285. DOI: 10.15406/jccr.2017.08.00285.

38. Centurión OA, Alderete JF, Torales JM, García LB, Scavenius KE, Miño LM, Sequeira OR, Chavez C. Electrophysiological Abnormalities of the
Atrial Myocardium and the Rubenstein Clinical Classification of Patients with Sinus Node Dysfunction. EC Cardiology 2018;5(11):846-57.

39. Acampa M, Lazzerini PE, Martini G. How to Identify Patients at Risk of Silent Atrial Fibrillation after Cryptogenic Stroke: Potential Role of $\mathrm{P}$ Wave Dispersion. J Stroke 2017; 19(2):239-41.

40. Fujimoto $\mathrm{Y}$, Yodogawa K, Takahashi K, Tsuboi I, Hayashi H, Uetake S, et al. Noninvasive evaluation of reverse atrial remodeling after catheter ablation of atrial fibrillation by $P$ wave dispersion. Heart Vessels 2017; 32(11): 1375-81.

41. Okutucu S, Oto A. P-wave dispersion in different clinical situations: Expanding list with resembling mechanisms. JRSM Cardiovasc Dis 2017; 6. https://doi.org/10.1177/20480040177203 69.

42. Kizilirmak $F$, Demir GG, Gokdeniz $T$, Gunes HM, Cakal B, Guler E, et al. Changes in electrocardiographic $P$ wave parameters after Cryoballoon ablation and their association with atrial fibrillation recurrence. Ann Noninvasive Electrocardiol 2016 ; 21(6): 580-7. 